\title{
ABSCESSO INTRABDOMINAL TARDIO PÓS COLECISTECTOMIA LAPAROSCÓPICA
}

\author{
LATE INTRABDOMINAL ABSCESS AFTER LAPAROSCOPIC \\ CHOLECYSTECTOMY
}

\author{
Júlio Cezar Uili Coelho, TCBC-PR ${ }^{1}$ \\ Ayrton Alves Aranha Junior ${ }^{2}$ \\ Lucas Mantovannni ${ }^{3}$
}

\section{INTRODUÇÃO}

Desde sua introdução no final da década de 80 , a colecistectomia laparoscópica tornou-se o tratamento de escolha para a colelitíase sintomática. Diversas são as complicações descritas relacionadas a este procedimento, cuja incidência varia de 2 a $6 \% \%^{1-3}$. A perfuração da vesícula biliar com extravasamento de cálculos pode ocorrer em até 20 - 30\% das colecistectomias ${ }^{1}$. Em alguns desses casos, um ou mais cálculos não são recuperados e podem permanecer na cavidade abdominal. Na maioria das vezes, o paciente permanece assintomático, mas em alguns casos, complicações podem advir dessa intercorrência ${ }^{2}$. O nosso objetivo no presente estudo é relatar a ocorrência de um caso de abscesso intrabdominal secundário a cálculo biliar cinco anos após colecistectomia laparoscópica.

\section{RELATO DO CASO}

Paciente feminina, de 75 anos de idade, relatou início de saída de secreção sero-sangüinolenta pelo umbigo em pequena quantidade, com início há cerca de um mês. Referiu mudança progressiva no aspecto da secreção, passando a purulenta nas duas últimas semanas. Relatava aumento da quantidade de drenagem aos esforços abdominais. Negava dor, febre, prurido, alterações de hábito intestinal ou urinário. Negava também icterícia, colúria ou acolia, bem como alergias ou transfusões sanguíneas. A paciente apresentava-se em tratamento para hipertensão arterial sistêmica. Tinha sido submetida à colecistectomia videolaparoscópica há cinco anos, tendo permanecido desde então assintomática. O exame anatomopatológico da peça cirúrgica evidenciou colecistite crônica e aguda.

Ao exame físico, a paciente apresentava-se normocorada, hidratada e anictérica. Os sinais vitais eram normais, bem como seu exame segmentar, com exceção a uma tumoração palpável em região infraumbilical, aparentemente intra-cavitária, de consistência firme, bem delimitada, móvel, indolor, com aproximadamente $5 \mathrm{~cm}$ em seu maior diâmetro.

Os exames laboratoriais, tanto hemograma, quanto metabólicos, eram normais. Ultra-sonografia abdominal demonstrou a presença de tumoração sólida de limites indefinidos, irregulares, medindo aproximadamente $56 \times 26 \mathrm{~mm}$ em região infra-umbilical, paramediana esquerda, intra-peritoneal. A lesão era composta por duas partes: a superior, com sombra acústica posterior, e a inferior, apenas ecogênica e heterogênea, com relação íntima com o fundo vesical (Figura-1).

1. Professor Titular do Departamento de Cirurgia da Universidade Federal do Paraná e Chefe do Serviço de Cirurgia Geral do Hospital Nossa Senhora das Graças.

2. Residente de Cirurgia Geral do Hospital Nossa Senhora das Graças.

3. Acadêmico de Medicina da Universidade Federal do Paraná. 


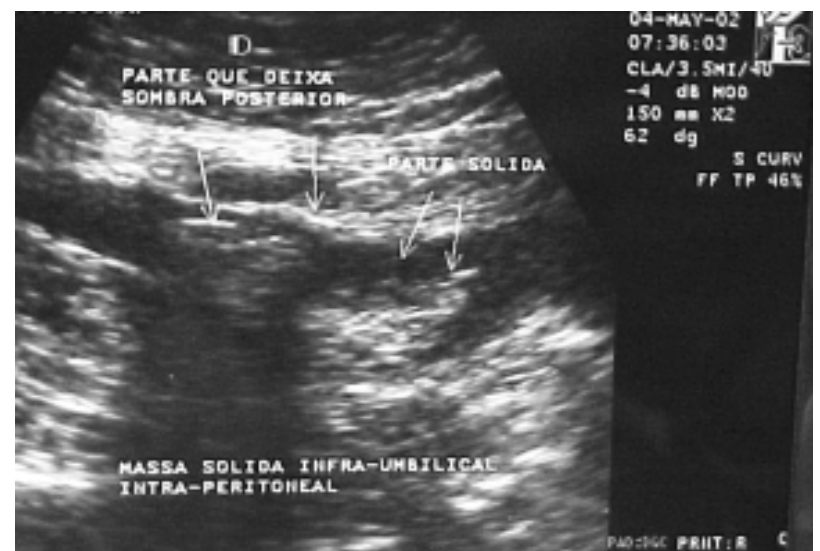

Figura 1 - Ultra-sonografia abdominal evidenciando tumoração sólida irregular com sombra acústica.

A paciente foi submetida a laparotomia infraumbilical, com saída de pequena quantidade de secreção purulenta após abertura da aponeurose. Abaixo da mesma observou-se intensa fibrose, formando um granuloma, no interior do qual encontravam-se dois cálculos biliares de 7 e $8 \mathrm{~mm}$ de diâmetro. Os cálculos foram retirados e a paciente evoluiu bem no pós-operatório, com parada da saída de secreção em sete dias.

\section{DISCUSSÃO}

A colecistectomia laparoscópica tornou-se rapidamente o tratamento de escolha da colelitíase sintomática, por apresentar poucas complicações, e permitir um tempo de hospitalização mais curto com retorno precoce do paciente às suas atividades diárias. Entretanto, o procedimento está associado a aumento do risco de perfuração da vesícula biliar, com conse- qüente extravasamento de bile e cálculos para a cavidade abdominal ${ }^{3}$.

O extravasamento de cálculos da vesícula biliar é relatado em até cerca de 20 a $30 \%$ das colecistectomias, podendo ocorrer durante o descolamento da vesícula do leito hepático ou durante a sua retirada pela incisão umbilical ${ }^{1}$. Normalmente esse tipo de acidente é percebido e os cálculos são removidos, sendo que na maioria das vezes em que algum cálculo permanece na cavidade abdominal, o mesmo permanece inerte, sem nenhum prejuízo para o paciente. Entretanto, algumas complicações têm sido descritas em pacientes em que um ou mais cálculos não foram recuperados ${ }^{4}$.

Läuffer et al. relataram uma revisão de 22 casos de cálculos biliares perdidos na cavidade abdominal em pacientes submetidos a colecistectomia laparoscópica ${ }^{4}$. A apresentação clínica incluiu abscessos em diferentes localizações, granulomas, fístulas, obstrução intestinal, migração dos cálculos para o canal femoral, pelve e ovário. No nosso meio, Castro et al. relataram um caso de eliminação de cálculos biliares através do trato urinário ${ }^{5}$. $\mathrm{O}$ caso por nós apresentado foi evidenciado pela drenagem espontânea de um abscesso intrabdominal através do umbigo cinco anos após a colecistectomia laparoscópica. Este achado confirma que cálculos deixados na cavidade abdominal podem manifestarse muitos anos após a colecistectomia, mesmo em pacientes que eram assintomáticos. Assim, todo esforço deve ser empreendido pelo cirurgião para retirar cálculos biliares extravasados da vesícula biliar durante a colecistectomia.

\begin{abstract}
Perforation of the gallbladder during laparoscopic cholecystectomy may be associated with intraperitoneal gallstone spillage. Several complications secondary to lost gallstones in the abdominal cavity have been described. We report a rare complication of abdominal abscess secondary to two gallstones left in the abdominal cavity. A 75-year-old female presented with spontaneous drainage of pus through the umbilicus five years after laparoscopic cholecystectomy. An ultrasonographic evaluation of the abdomen revealed a solid mass of $56 \times 26 \mathrm{~mm}$ of diameter, with acoustic shadow, localized distal to the umbilicus. At laparotomy, an abscess with two biliary calculi was drained. The patient had good recovery, with no complication.
\end{abstract}

Key words: Cholecystectomy laparoscopic; Abdominal abscess. 


\section{REFERÊNCIAS}

1. Warren CW, Wyatt JI. - Gallstones split at laparoscopic cholecystectomy: a new case of intraperitoneal granulomas. J Clin Pathol, 1996, 49(1): 84-85.

2. Vdlamudi G, Graebe R, Khoo M, Schinella R. - Gallstones implanting in the ovary: A complication of laparoscopic cholecystectomy. Arch Pathol Lab Med, 1997, 121(2): 155-158.

3. Merchant SH, Haghir S, Gordon GB. - Granulomatous peritonitis after laparoscopic cholecystectomy mimicking pelvic endometriosis. Obstet Gynecol, 2000, 96(5 Pt 2): 830-831.

4. Läuffer JR, Krähenbühl L, Baer HU, et al. - Clinical manifestations of lost gallstones after laparoscopic cholecystectomy: a case report with review of literature. Surg Laparosc Endosc, 1997, 7(2): 103-112.

5. Castro MG, Alves AS, Oliveira CA, et al. - Elimination of biliary stones through the urinary tract: a complication of the laparoscopic cholecystectomy. Rev Hosp Clín Fac Med São Paulo, 1999, 54(6): 209-212.

Endereço para correspondência:

Dr. Júlio Coelho

Rua Bento Viana, 1140 - Ap. 2202

80240-110 - Curitiba - PR

Tel/Fax.: + $5541322-3789$ 